\section{Zur Frage der}

Resonanz bei Atomzertrümmerungsprozessen.

Die Frage, ob bei der künstlichen Zertrümmerung Resonanzerscheinungen auftreten, wurde in letzter Zeit mehrfach vom experimentellen und theoretischen Standpunkt aus behandelt. Pose fand beim Aluminium und neuerdings auch beim Fluor Gruppen von Zertrümmerungsprotonen, welche nur von $\alpha$-Teilchen ganz bestimmter Energie hervorgebracht sein sollen. Gelegentlich von Versuchen, die der Verf, über die Zertrümmerung von Aluminium mit Polonium- $\alpha$-Strahlen angestellt hat, wurde auch die Frage der Resonanz geprüft. Die zertrümmernden $\alpha$-Strahlen wurden hierbei durch Kohlensäure von entsprechenden Drucken verschieden stark abgebremst und die Intensität der ausgelösten H-Strahlen für zwei passend gewählte Reich- weitestellen gemessen. Die Messungen ergaben keine Bestätigung der Poseschen Resultate. Es zeigte sich eine kontinuierliche Abnahme der Intensität der $\mathrm{H}$ Teilchen mit abnehmender $\alpha$-Energie. Eine ausführliche Darstellung folgt in der Zeitschrift für Physik.

Berlin-Dahlem, Kaiser Wilhelm-Institut für Chemie, Physikalisch-radioaktive Abteilung, den 6. Dezember r93. E. STEUDEL.

Berichtigung.

Die Ansicht, daß sich SugIuras Versuche über Protonenbeugung als Kreuzgitterinterferenzen auffassen lassen, läßt sich nicht aufrecht erhalten. Die in Heft 47, S. 95I erwähnten scharfen Grenzen der Intensitatsverteilung finden sich nämlich in der durchgehenden, nicht in der reflektierten. Strahlung.

Berlin, den 12. Dezember I93x. M. v. LAuE.

\title{
Besprechungen.
}

GOEBEL, KARL, Organographie der Pflanzen. 2. Teil. Bryophyten-Pteridophyten. 3. umgearbeitete Auflage. Jena: Gustav Fischer r93o. X, S. 643-1378 und $850 \mathrm{Abb} . \quad 17 \times 26 \mathrm{~cm}$. Preis geh. RM 35.-, geb. RM 37.-

Dem in den Naturwiss. I7, H. $49,969 \mathrm{f},(1929)$ angezeigten I. Teil der 3. Auflage von Goebels Organographie ist nun der 2. Teil dieser Auflage gefolgt, der eine kritische Zusammenfassung unseres Wissens uber die Organographie der Bryophyten und Pteridophyten bis in die Gegenwart hinein bietet. Es wird nicht viele Darstellungen eines großen Wissenschaftsgebietes geben, die in so weitem Umfang auf Einzelforschungen des Bearbeiters der Zusammenfassung selbst beruhen, und deren Geist in so hohem Maße von diesem Bearbeiter bestimmt ist, wie dies von GoEBELS Organographie, und vor allem von dem vorliegenden Teil gilt. Was über die grundsätzliche Bedeutung des großen Werkes bei der Besprechung des r. Teiles gesagt wurde, trifft ebenso wie die Feststellung tuber die sorgfältige Erneuerungsarbeit für die neue Auflage des 2. Teiles zu. Wiederum ist durch alle Kapitel hindurch die kritisch prüfende und bessernde Hand $z u$ spüren, die Uberholtes beseitigt, durch eigene und fremde Forschung Neugefundenes unter sorgsamer Berüclksichtigung der Literatur bis zur Gegenwart an der richtigen Stelle einfügt und vielfach gröBere oder kleinere Abschnitte völlig neu formt. Schon die II9 neuen Abbildungen bezeugen dies aufs deutlichste. Wenn die GesamtseitenzahI des Bandes gegenüber dem der 2. Auflage nur um 39 stieg (von denen 8 auf die Bryophyten, 3I auf die Pteridophyten fallen), so besagt dies nichts über den tatsächlichen Umfang der Änderungen und neuen Abschnitte, da diese zum Teil ältere ersetzen, und ferner, besonders bei den Farnen, die Abschnitte über die fossilen Formen und die (vorwiegend kritischen, ja skeptischen) Erörterungen über phylogenetische Probleme sowie über anatomische Fragen (z. B. \& 3 der Einleitung $z u$ den Pteridophyten in der. 2. Auflage) gestrichen sind, und außerdem durch geschickte drucktechnische Anordnung besonders der Abbildungen an Raum gespart wurde. An AuBerlichkeiten ist zu vermerken, daß die Inhaltsübersicht durch Weglassen der Stichwörter erheblich kürzer gefaßt ist. Ein kleiner Schönheitsfehler ist insofern stehen geblieben, als bei den Bryophyten die Paragraphen durch alle drei Abschnitte mit ihren Kapiteln durchnumeriert werden von I -72 , während bei den Pteridophyten jedes Einzelkapitel der fünf Abschnitte seine eigene Paragraphennumerierung hat.

Da auch dieser für einen großen Stoffkreis wie seine wissenschaftliche Forschungsweise grundlegende Teil des in keinet botanischen Fachbücherei fehlenden Werkes als unerschöpfliche Fundgrube jedem Botaniker von seinen früheren Auflagen her vertraut ist, so genügen hier Hinweise auf die wissenschaftlich wesentlichstea Änderungen der neuen Auflage.

Bei den Bryophyten enthält der I. Abschnitt ,Einleitung" einen neuen $\$ 7$, ,Einteilung der Bryophyten" (S. 687f.), der an die Stelle der einleitenden Bemerkungen im $\$ 2$ der 2. Auflage tritt. Die Selbständigkeit der Andreaeaceen, Sphagnaceen wnd Anthocerotaceen gegenüber den Laubmoosen i. c. S. und Lebermoosen i. e. S. wird stärker betont, und hervorgehoben, daß die verschiedene Verteilung von Eigenschaften darauf hinweise, ,daß die Bryophyten eine polyphyletische Gruppe darstellen, deren ursprünglich gleiche ,Entwicklungsmöglichkeiten' dann auf die verschiedenen sich a bspaltenden Gruppen verschieden verteilt wurden ". Dabei wird die Unterscheidung von 5 oder von 2 Gruppen als reine Zweckmäßiglkeitsfrage bebandelt und die alte HEDw IGsche Teilung in Lebermoose und Laubmoose ,immer noch für berechtigt" erklärt. Eine klare Ubersicht über GoEBELs Auffassung der natürlichen Gruppen der Lebermoose gibt der neue $\$$ 4oa (S. 910 bis 9r3) am Ende des Lebermoosabschnitts, der nur ân Berlich durch Herauslösung aus dem 7 . Kapitel, ,Die Sporenkeimung", hätte besser gekennzeichnet werden können; eine entsprechende Darstellung der natürlichen Gruppen der Laubmoose scheint der Verf. nicht für notwendig gehalten zu haben. Im I. Kapitel des Lebermoosabschnittes, ,Die Gestaltung der Vegetationsorgane", findet sich im \& Io (Utbergang vom Thallus zum beblätterten Sproß) eine erweiterte Darstellung der Verhältnisse bei Petalophyllum mit neuen Abbildungen $(703,704)$, die eine Modifikation der ursprünglichen LEITGEBschen Auffassungsweise der hier auftretenden Lamellen darstellt, welche nach neuen Untersuchungen GoEBELS nur einem Teil des Blattes entsprechen (S. 7I $3 \mathrm{f}$ ). Der \& I2 (Beziehungen zwischen thalloser und folloser Ausbildung des Vegetationskörpers) erhält Erweiterungen dadurch, daß GOEBEL inzwischen die Entwicklungsgeschichte von Schiffneria (und die Ausbildung der Amphigastrien) untersucht hat, deren Ergebnisse seine bisherige Auffassung der morphologischen Verhältnisse dieser Gattung stützen. Im 2. Kapitel, ,Die anatomische Gliederung“, ist im $\$ 16$ (der Bau des Marchantiathallus) die Darstellung der Luftkammern auf Grund neuer Untersuchungen unter Vermehrung der Abbildungen exweitert. Im 3. Kapitel ,.,Die Beziehungen der Organbildung $z u$ den Lebensbedingungen", scheint mir die 